\title{
Trends in the use of second trimester maternal serum screening from 1991 to 2003
}

Peter A. Benn, $P h D^{1}$, Min Fang, MD, $P h D^{1}$, and James F.X. Egan, $M D^{2}$

\begin{abstract}
Purpose: To identify trends in the utilization of second trimester maternal serum screening, follow-up amniocenteses, and the detection of Down syndrome-affected pregnancies between 1991 and 2003. Methods: We reviewed all triple and quadruple maternal serum screening tests referred to the University of Connecticut screening laboratory from women with singleton pregnancies. For each calendar year, the total number of tests, proportion to women aged 35 or older, number of follow-up amniocenteses, and the number of prenatally and postnatally diagnosed Down syndrome cases were recorded. Results: A total of 109,469 women received screening. In 1991, the proportion of older women who received screening was $58 \%$ of that present in the Connecticut population but by 2003 this had increased to 83\% $(P<0.001)$. In Down syndrome screen-positive pregnancies, there was no significant change in the rate of amniocentesis utilization (average $73 \%$ ), but in false-positives, there was a decline from $70 \%$ in 1991 to $27 \%$ in $2003(P<0.001)$. Conclusion: Increased use of maternal serum screening by older women, use of second trimester ultrasound, and improvements in screening methodology have resulted in sharply reduced numbers of amniocenteses in unaffected pregnancies. Genet Med
\end{abstract}

2005:7(5):328-331.

Key Words: prenatal, screening, Down syndrome, trimester, utilization

The incidence of autosomal trisomy in livebirths is strongly dependent on maternal age. ${ }^{1}$ Because of this well-recognized association, special consideration is given to the provision of prenatal cytogenetic testing to women aged 35 or more at delivery. Professional guidelines from the American College of Medical Genetics (ACMG) ${ }^{2}$ and the American College of Obstetrics and Gynecology (ACOG) ${ }^{3}$ state that invasive diagnostic testing (prenatal chromosome analysis through amniocentesis or chorionic villus sampling, CVS) should be offered to women aged 35 or older (advanced maternal age, AMA). Second trimester multiple marker screening is recommended for women "unless amniocentesis is indicated based on their history and/or age or they have elected to have first trimester screening and/or CVS."2 These age-based guidelines have remained largely unaltered despite the proven efficacy of second trimester maternal serum screening, ${ }^{4-6}$ widespread use of ultrasound to identify fetal structural abnormalities or markers associated with aneuploidy, ${ }^{7}$ demonstrated performance of first trimester screening, ${ }^{8,9}$ and the potential shown for combinations of these approaches. ${ }^{10,11} \mathrm{~A}$ recent alternative set of

\footnotetext{
From the ${ }^{I}$ Division of Human Genetics, Department of Genetics and Developmental Biology, and the ${ }^{2}$ Department of Obstetrics and Gynecology, University of Connecticut Health Center, Farmington, Connecticut.

Peter A. Benn, PhD, Division of Human Genetics, Department of Genetics and Developmental Biology, University of Connecticut Health Center, 263 Farmington Avenue, Farmington, CT 06030-6140.

Received: January 10, 2005.

Accepted: February 8, 2005.
}

DOI: 10.1097/01.GIM.0000162875.36352.A9 guidelines issued by the International Down syndrome Screening Group (IDSSG) indicated that the use of maternal age as a sole criterion for aneuploidy risk assessment was no longer justified. ${ }^{12}$ The IDSSG guidelines called for the use of the best possible estimate of risk for every patient and provided specific recommendations for the screening protocols that should be used.

In this study, we report on steady improvements in the overall effectiveness of second trimester serum screening and comment on the appropriateness of the most recent version of the ACMG guidelines.

\section{MATERIALS AND METHODS}

We reviewed all maternal serum screening tests performed on singleton pregnancies that were referred to the University of Connecticut Health Center Human Genetics Laboratories from September 1991 to December 2003. Initial studies were based on maternal serum alpha-fetoprotein (MS-AFP), unconjugated estriol (uE3), and human chorionic gonadotropin (hCG). In April 1999, this screening test was expanded to include inhibin-A (INH-A). A second trimester cut-off of 1:270 was used to define screen-positive pregnancies. A first trimester screening protocol was not in place during the time interval covered by the study. Second trimester ultrasound to detect fetal structural abnormalities and fetal markers associated with aneuploidy was available throughout this interval with postserum screening risks modified from January 1996 onwards.

The methods used to gather pregnancy outcome information have been previously described. ${ }^{13}$ Follow-up data in- 
cluded utilization of amniocentesis, cytogenetic results, and the presence of Down syndrome or other aneuploidy identified at birth. Expected numbers of Down syndrome births were based on the 8 -series curve of Bray et al. ${ }^{14}$ and the number of affected second trimester pregnancies derived by applying an adjustment for in utero survival. ${ }^{15}$ The proportion of births to women aged 35 or more in Connecticut were obtained from birth certificate data. ${ }^{16}$

Chi-squared tests were carried out using SPSS for Windows (SPSS Inc., Chicago, IL). A value of $P<0.05$ was considered statistically significant.

\section{RESULTS}

Between September 1991 and December 2003, maternal serum screening was provided to a total of 109,469 women with singleton pregnancies. Overall, $12.9 \%$ of the screened population was aged 35 or older at delivery. Figure 1 shows the annual proportion of screened women who were aged 35 or older at delivery, compared to the proportion of AMA mothers for in Connecticut births. The growth in the proportion of AMA women who received screening approximately paralleled the increase in older mothers in the whole population. In 1991, the proportion of AMA women who received screening was $58 \%$ of that present in the whole population but by 2003 this had increased to $83 \%$. The proportion of AMA women receiving serum screening, standardized for the increasing numbers of these women in the Connecticut population, was statistically significant $(P<0.001)$.

Of the 95,345 women under 35 years at delivery, 3,980 $(4.2 \%)$ were screen-positive for fetal Down syndrome. Of these screen-positive women, 1,973 accepted amniocentesis and therefore the net rate of amniocentesis in younger women was $2.1 \%$. For the 14,124 women age 35 or older, 3,287 (23.3\%) were screen-positive and 1,391 received amniocentesis resulting in an overall rate of amniocentesis of $9.8 \%$.

Figure 2 shows the annual changes in the utilization of amniocentesis by these two groups of women. Between 1991 and 2003 , there was a marked reduction in the utilization of am-

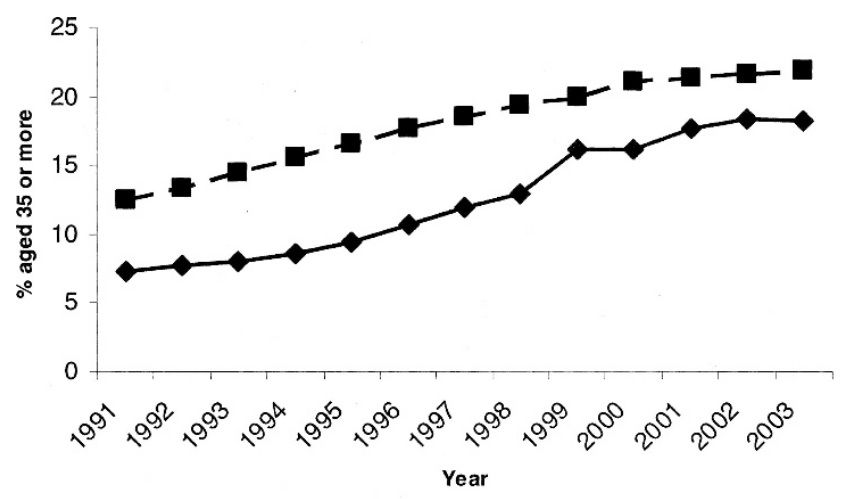

Fig. 1. Proportion of women in Connecticut who are of advanced maternal age (top line) and the proportion of women who received screening and are of advanced maternal age (bottom line). niocentesis by both groups. For all ages combined, the proportion of screen-positive women who underwent amniocentesis in 1991 was $70 \%$ but by 2003 this had declined to $27.7 \%$. The difference in the rate of utilization of amniocentesis by younger versus older screen-positive women was statistically significant in earlier years of the study (1991-1993, $1995-1996)(P<0.05)$ but not significant in all other years.

Based on the maternal ages of the 109,469 women screened, there should have been 217 second trimester Down syndrome fetuses present in the screened population. This probably represents an overestimate of the actual number present because up to $11 \%$ of Down syndrome second trimester pregnancies are ascertained through abnormal ultrasound or increased risk due to family history, without provision of maternal serum screening. ${ }^{17}$ Based on our follow-up data, we could account for 203 cases (94\% of the upper estimate of 217 cases). We therefore judged ascertainment of affected pregnancies to be substantially complete. Table 1 summarizes the detection rates, false-positive rates, and overall impact of the screening based on this follow-up data. Figure 3 shows the detection rates and false-positive rates for each year of the study.

A further analysis of amniocentesis rates separating true positive cases from false-positives showed that the overall reduction in amniocentesis utilization was confined to false-positive cases (Fig. 4). In affected pregnancies, there was no significant change in the rate of amniocentesis (average 73\%) $(P>$ $0.05)$, but in false-positives, the decline from $70 \%$ in 1991 to $27 \%$ in 2003 was significant $(P<0.001)$. For the first 3 years of the study (1991-1994), there was an average of one Down syndrome diagnosis for every 69 amniocenteses and for the last 3 years (1999-2003), there was one affected pregnancy in every 20 amniocenteses.

\section{DISCUSSION}

In this report, we document improved efficacy of second trimester Down syndrome screening when evaluated from the perspective of the number of affected pregnancies diagnosed relative to the number of amniocenteses performed. These ob-

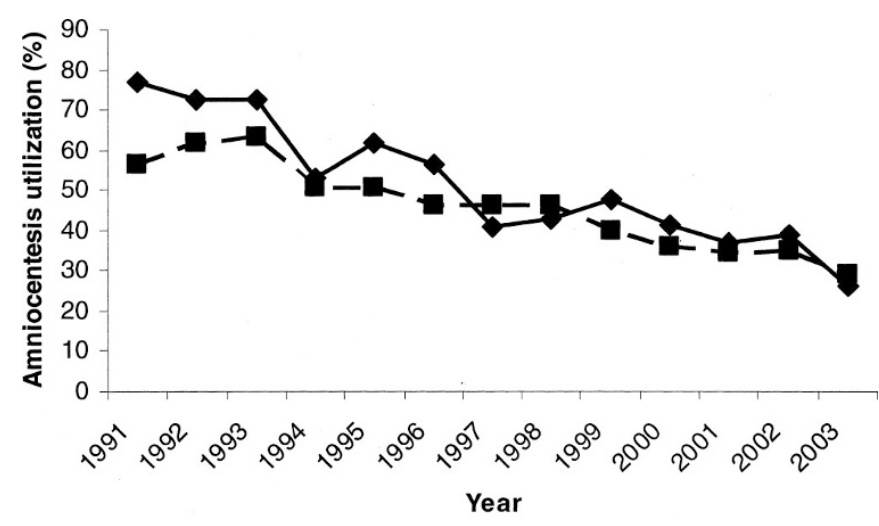

Fig. 2. Decline in the use of amniocentesis among screen-positive women aged 35 or older $(\square)$ and by younger women $(\bullet)$. 
Table 1

Efficacy of the second trimester serum screening for Down syndrome using detection, false-positives, and amniocentesis utilization as measures

\begin{tabular}{lccc}
\hline & \multicolumn{3}{c}{ Maternal age } \\
\cline { 2 - 4 } & $<35$ & $\geq 35$ & All \\
\hline Down syndrome & & & \\
Screen-positive (\%) & $72(62.6)$ & $77(88.5)$ & $149(73.5)$ \\
Screen-negative (\%) & $43(37.4)$ & $10(11.5)$ & $54(26.5)$ \\
Total & 115 & 87 & 203 \\
Amniocenteses (\%) & $47(40.9)$ & $57(65.5)$ & $104(51.2)$ \\
Unaffected & & & \\
Screen-positive (\%) & $3,912(4.1)$ & $3,213(22.9)$ & $7,125(6.5)$ \\
Screen-negative (\%) & $91,318(95.9)$ & $10,824(77.1)$ & $102,141(93.5)$ \\
Total & 95,230 & 14,037 & 109,266 \\
Amniocenteses (\%) & $1,926(2.0)$ & $1,334(9.5)$ & $3,260(3.0)$ \\
\hline
\end{tabular}

Numbers and rates reflect adjustment for unrecognized Down syndrome cases that would be expected to spontaneously abort between the second trimester and term.

servations confirm and extend trends seen in referrals to our cytogenetics laboratory. ${ }^{17}$ The improvement is probably attributable to three factors: provision and acceptance of maternal serum screening by older women; the use of second trimester ultrasound as an adjunct to serum screening; and improvements in serum screening such as the incorporation of the INH-A in testing.

When maternal serum screening is provided to older women, both the detection rate and the false-positive rate are increased, but overall, the screening is more effective with a higher positive predictive value (true positives divided by true plus false-positives) compared to that achieved for younger women. ${ }^{18}$ Consistent with theoretical expectations for this screening, ${ }^{19}$ we observed an approximately $88 \%$ detection rate and $22.9 \%$ false-positive rate for women aged 35 or more. For our screened population, if amniocentesis had been provided to all serum screen-negative women aged 35 or more, there would have been 10,837 additional amniocenteses, 10 additional Down syndrome affected pregnancies identified, and an estimated 65 additional fetal losses. ${ }^{20}$

Retaining maternal age alone as an appropriate indication for offering amniocentesis has been justified on the basis that not all cases of Down syndrome will be identified through screening and that there are other maternal age-associated fetal aneuploidies that are not identified. The second trimester quadruple test is expected to identify $90 \%$ of Down syndrome and $87 \%$ of trisomy 18 pregnancies in women aged 35 or more. ${ }^{19}$ The second trimester group risk for fetal chromosome abnormality in AMA screen-negative women is therefore approximately $1.0 \%$ of which $0.4 \%$ are balanced translocations, 47,XXX and 47,XYY karyotypes..$^{21}$ Although prenatal diagnosis of these latter karyotypes may be helpful for some parents, this has to be weighed against the approximately $0.6 \%$ risk of an amniocentesis procedure-related risk of fetal loss. ${ }^{20}$ Balanc- ing the risk of aneuploidy against risk of a procedure-related fetal loss is particularly important for older women who show declining fertility. We have advocated the use of serum screening in older women because of these considerations, the increasing numbers of older mothers in the United States, ${ }^{22}$ and because maternal serum screening is cost effective. ${ }^{23}$

Women who are screen-positive for Down syndrome will generally receive an ultrasound exam to look for the presence or absence of fetal structural abnormalities or markers associated with aneuploidy. It has become common practice to reduce the postserum screening risk if no anomalies or markers are present, typically by $50 \%$ and to increase risks using specific likelihood ratios if there is evidence suggestive of aneuploidy. ${ }^{24}$ These approaches can be expected to reduce the effective falsepositive rate by over $50 \%{ }^{25}$ but concerns have been expressed that the detection rate may also be diminished. ${ }^{26}$ Our observation that the amniocentesis rate declined in false-positive cases but not in women with affected pregnancies implies that the use of second trimester ultrasound to modify risk is in fact highly beneficial. The impact of second trimester ultrasound to help identify Down syndrome and other aneuploidies was not addressed in the ACMG guidelines.

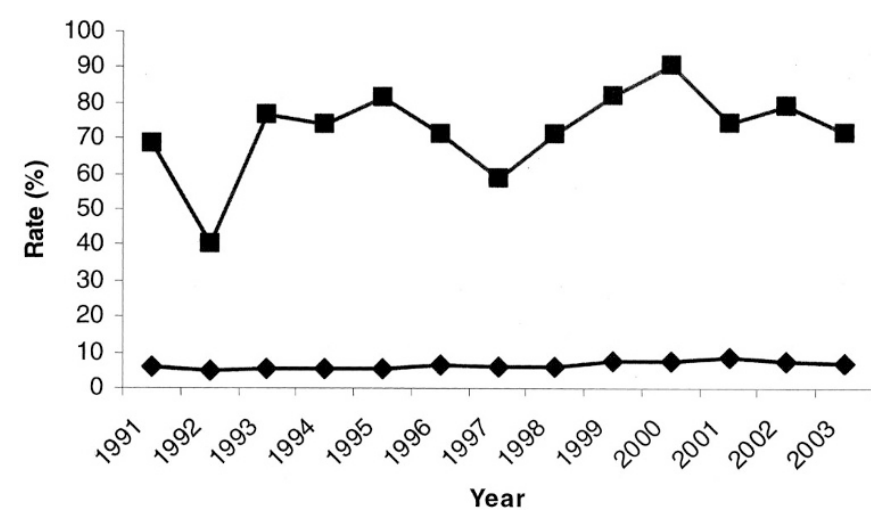

Fig. 3. Detection rate (top line) and false-positive rate (bottom line) for second trimester maternal serum screening.

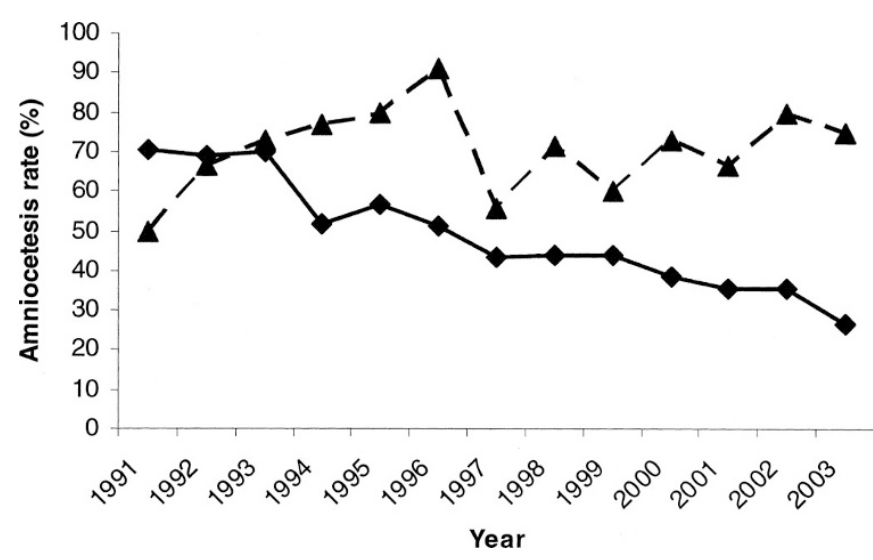

Fig. 4. Amniocentesis utilization among screen-positive women with affected $(\boldsymbol{\Lambda})$ and unaffected $(\diamond)$ pregnancies. 
The advantage of adding INH-A to second trimester serum screening may also have been under appreciated. Adding INH-A to the triple test for our population resulted in an improvement in the detection rate from $79.3 \%$ to $83.8 \%$ and a reduction in the false-positive rates from $10.4 \%$ to $9.9 \% .^{5}$ These gains may be viewed as relatively modest, but this perspective does not take into consideration an additional advantage associated with the inclusion of INH-A. In affected pregnancies, the median risk is increased from 1:57 to $1: 30,{ }^{27}$ and because the reported risk figure is an important determinant in amniocentesis utilization for screen-positive women, ${ }^{28}$ there are likely to be additional cases of Down syndrome prenatally diagnosed. Conversely, in unaffected pregnancies, use of INH-A results in greater reassurance with median risk reduced from 1:3400 to 1:6000. The ACMG guideline does not take a position on the use of INH-A.

The purpose of prenatal screening is to maximize reproductive options available, ${ }^{29}$ and this goal is best achieved by providing each woman with the best possible estimate of her personal risk for fetal aneuploidy. ${ }^{30}$ The current ACMG guideline ${ }^{2}$ may have the unfortunate effect of limiting older women's access to screening. Guidelines for the United States need to be flexible to reflect individual patient preferences, differences in the gestational age when women first seek prenatal care, regional differences in the availability of screening tests, and the need to protect against unjustified medical liability claims. We urge the American College of Medical Genetics Practice Committee to consider adopting policies similar to those outlined in the IDSSG statement. ${ }^{12}$

\section{References}

1. Hook EB. Prevalence, risks and recurrence. In: Brock DJH, Rodeck CH, FergusonSmith MA, editors. Prenatal Diagnosis and Screening. Edinburgh: Churchill Livingston, 1992

2. Driscoll DA for the Professional Practice and Guidelines Committee. Second trimester maternal serum screening for open neural tube defects and aneuploidy. Genet Med 2004;6:540-541.

3. American College of Obstetricians and Gynecologists. ACOG Pract Bull. May 2001; 27.

4. Cuckle H. Established markers in second trimester maternal serum. Early Hum Develop 1996;47:S27-S29.

5. Benn PA, Fang M, Egan JFX, Horne D, Collins R. Incorporation of Inhibin-A in second-trimester screening for Down syndrome. Obstet Gynecol 2003;101:451-454.

6. Wald NJ, Huttley WJ, Hackshaw AK. Antenatal screening for Down's syndrome with the quadruple test. Lancet 2003;361:835-836.

7. Shipp TD, Benacerraf BR. Second trimester ultrasound screening for chromosomal abnormalities. Prenat Diagn 2002;22:296-307.

8. Bindra, Heath V, Liao A, Spencer K, Nicolaides KH. One-stop clinic for assessment for risk for trisomy 21 at 11-14 weeks: a prospective study of 15,030 pregnancies. Ultrasound Obstet Gynecol 2002;20:219-225.
9. Wapner R, Thom E, Simpson JL, Pergament E, Silver R, Filkins K et al; First Trimester Maternal Serum Biochemistry and Fetal Nuchal Translucency Screening (BUN) Study Group. First-trimester screening for trisomies 21 and 18. N Engl J Med 2003; 349:1405-1413.

10. Wald NJ, Watt HC, Hackshaw AK. Integrated screening for Down's syndrome on the basis of tests performed during the first and second trimesters. N Engl J Med 1999;341:461-467.

11. Wright D, Bradbury I, Benn P, Cuckle H, Ritchie K. Contingent screening is an efficient alternative to non-disclosure sequential screening. Prenat Diagn 24:762766.

12. Ferguson-Smith M, Aitken D, Arbuzova S, Benn P, Canick J, Cuckle H et al. Down syndrome screening: a position statement from the scientific committee of the international Down syndrome screening group, 2004. Available at: http://www.leeds.ac.uk/idssg/position\%20statement.htm. Accessed January 10, 2005.

13. Benn PA. Preliminary evidence for associations between second trimester human chorionic gonadotropin and unconjugated estriol levels with pregnancy outcome in Down syndrome pregnancies. Prenat Diagn 1998;18:319-324.

14. Bray I, Wright DE, Davies C, Hook EB. Joint estimation of Down syndrome risk and ascertainment rates: a meta-analysis of nine published data sets. Prenat Diagn 1998; 18:9-20.

15. Benn PA, Egan JFX. Survival of Down syndrome in utero. Prenat Diagn 2000;20: 432-439.

16. National Center for Health Statistics. Vital statistics of the United States: 1991-2001. Natality Data Set. Hyattsville, MD.

17. Benn PA, Egan JFX, Fang M, Smith-Bindman R. Changes in the utilization of prenatal diagnosis. Obstet Gynecol 2004;103:1255-1260.

18. Benn PA. Advances in prenatal screening for Down syndrome: I general principles and second trimester testing. Clinica Chimica Acta 2002;323:1-16.

19. Benn PA, Ying J, Beazoglou T, Egan JFX. Estimates for the sensitivity and falsepositive rates for second trimester serum screening for Down syndrome and trisomy 18 with adjustment for cross-identification and double-positive results. Prenat Diagn 2001;21:46-51.

20. Seeds JW. Diagnostic mid trimester amniocentesis: how safe? Am J Obstet Gynecol 2004;191:608-616.

21. Ferguson-Smith MA, Yates JRW. Maternal age specific rates for chromosome aberrations and factors influencing them: report of a collaborative European study on 52,965 amniocenteses. Prenat Diagn 1984;4S:5-44.

22. Egan JFX, Benn P, Borgida AF, Rodis JF, Campbell WA, Vintzileos AM. Efficacy of screening for Down syndrome in the United States from 1974 to 1997. Obstet Gynecol 2000;96:979-985.

23. Beazoglou T, Heffley D, Kyriopoulos J, Vintzileos A, Benn PA. Economic evaluation of prenatal screening for Down syndrome in the USA. Prenat Diagn 1998;18:12411252.

24. Egan JFX, Kaminsky LM, DeRoche ME, Barsoom MJ, Borgida AF, Benn PA. Antenatal Down syndrome screening in the U. S. in 2001: A survey of maternal-fetal medicine specialists. Am J Obstet Gynecol 2002;187:1230-1234.

25. Pinette MG, Egan JFX, Wax JR, Blackstone J, Cartin A, Benn PA. Combined sonographic and biochemical markers for Down syndrome screening. J Ultrasound Med 2003;22:1185-1190.

26. Haddow JE, Palomaki GE, Knight GJ, Canick JA. Prenatal Screening for Major Fetal disorders. The Foundation for Blood Research handbook, II: Screening for Down syndrome. Maine: Foundation for Blood Research, 1998.

27. Benn PA, Fang M, Egan JFX, Horne D, Collins R. Incorporation of Inhibin-A in second-trimester screening for Down syndrome: Reply to Hallahan et al. Obstet Gynecol 2003;102:413-414.

28. Chen J, Heffley D, Beazoglou T, Benn P. Utilization of amniocentesis by women screening positive for Down syndrome on the second-trimester triple test. Commun Genet 2000;3:24-30.

29. Rowley PT. Genetic screening: marvel or menace? Science 1984;225:138-144.

30. Benn PA. Improved antenatal screening for Down's syndrome. Lancet 2003;361: $794-795$. 